\title{
Assessment of Effects of Different Mydriatics on Choroidal Thickness by Examining Anterior Chamber Parameters
}

\author{
Fahmy RM ${ }^{1 *}$ and Hadeel Alamer ${ }^{2}$ \\ ${ }^{1}$ Department of Ophthalmology, Faculty of Medicine, Cairo university, Egypt. \\ ${ }^{2}$ Department of Optometry and Vision Sciences, Saudi Arabia
}

*Corresponding author: Fahmy RM, Department of Ophthalmology, Faculty of

Medicine, Cairo university, Egypt.

Received Date: November 23, 2018

Published Date: January 11, 2019

\begin{abstract}
Background: Mydriatics drops have a broad range of applications in ocular examination and treatment. After administration, many changes can occur in the different parts of the eye including the choroidal layer which is a vascular layer localized between the outer sclera and inner retinal layers.

Purpose: To study effects of different mydriatics on choroidal thickness (CHT) by examining anterior chamber parameters (corneal thickness, corneal volume, corneal curvature, anterior chamber depth (ACD), anterior chamber volume (ACV) and anterior chamber angle (ACA).

Materials and methods: A total of 166 eyes of 83 students and employee aged 18-40 years old were enrolled in this crosssectional study. The participants were divided randomly into 3 groups based on application of drops (tropicamide group, phenylephrine group and cyclopentolate group). All subjects underwent a full ophthalmic examination involving; visual acuity and refraction, Slit- lamp biomicroscopy, Fundus examination, IOP measurement using noncontact tonometry, anterior chamber parameters measurement using Pentacam Rotating Scheimpflug Camera and the choroidal thickness measurement by Optical coherence tomography OCT.
\end{abstract}

Result: Decrease in the peri-temporal, inner-temporal, sub foveal, inner-nasal and peri-nasal choroidal thickness after drops instillation in all groups were found to be significant ( $p=0.000, p=0.000, p=0.000, p=0.000$ and $p=0.000$ respectively), while increase in the anterior chamber depth was found to be significant with all mydriatics $(p=0.00)$.

Conclusion: The study showed that while cyclopentolate, tropicamide, and phenylephrine cause a decrease in choroidal thickness, they also lead to increase in anterior chamber depth.

Keywords: Mydriatics; Choroidal thickness; Anterior chamber parameters; Pentacam; OCT

Abbreviations: Cht Peri_Tem_PRE, POST: Peri-Temporal Choroidal Thickness Pre-\& Post Drop Instillation; Cht_Inner_Tem_PRE, POST: Inner-Temporal Choroidal Thickness Pre-\& Post Drop Instillation; Cht_Subfoveal_PRE, POST: Sub Foveal Choroidal Thickness Pre-\& Post Drop Instillation; Cht_Inner_Nas_PRE, POST: Inner-Nasal Choroidal Thickness Pre-\& Post Drop Instillation; Cht_Peri_ Nas_PRE, POST: Peri-Nasal Choroidal Thickness Pre-\&Post Drop instillation

\section{Introduction}

Mydriatic eye drops have a wide range of uses in ocular examination and management performing pupillary dilatation and or cycloplegia. Clinically, mydriatics are utilized to induce cycloplegia for refractive errors diagnosis, to obtain pupillary dilatation for fundus biomicroscopic examination, surgical procedures, and angiography, or to avoid synechia and adhesions in cases of chronic uveitis. After instillation of mydriatics, several changes manifest in

the iris thickness and volume as well as anterior chamber angle and depth associated with pupil dilation [1-3].

Choroid is a dark brown, highly vascular layer situated between the sclera and retina. It extends from the ora serrata up to the aperture of the optic nerve in the sclera. Its thickness can't be assessed accurately because of its extreme vascularity. It is thicker posteriorly $(1 \backslash 4 \mathrm{~mm})$ than anteriorly $(1 \backslash 10 \mathrm{~mm})$. The choroid is 
adherent to the retina at two sites: at the margin of the optic nerve and at the scleral spur. It has many prime functions including nutrition of outer retinal layers [4,5]. Better comprehension of the choroidal framework may participate to the diagnosis and management of various ocular diseases. 3D OCT-2000 Spectral Domain OCT is a high-resolution, cross-sectional imaging technique that allows detailed assessment of retinal and choroidal thickness. With OCT, exact localization of pathologic features can be visualized in segmentation maps. Therefore, it becomes an important clinical and research tool for the diagnosis, follow up, treatment, and assessment of new treatment modalities for all diseases that affect the posterior pole of the eye. In many studies using OCT, it has been proved that choroidal thickness is influenced by various diseases like age related macular degeneration, malignant myopia and central serous chorioretinopathy [6-9].

The choroid as a part of the uveal tract as the iris may exhibit confirmed changes after mydriatic drops instillation. There has been restricted number of studies measuring choroidal thickness after mydriatic use. These studies revealed discordant results after measurements with efficient pupillary dilatation in healthy individuals, showing no change or thinning in choroid [10]. In our study, we have examined the hypothesis that the choroidal thickness will change with the application of the 3 commonly used mydriatics (cyclopentolate, tropicamide, and phenylephrine) and we examined whether these potential changes can be estimated through measurement of anterior chamber parameters.

\section{Methods}

The study was approved by the concerned Ethical Committee and informed consent followed the tenets of the Declaration of Helsinki. Subjects with best corrected visual acuity 20/ 25 or better, with refractive error $(\leq \pm 6.50 \mathrm{SE})$ were eligible for this study. The exclusion criteria included those subjects who had a history of retinal diseases, ocular injury or surgery, any reasons for poor image quality of OCT such as unstable fixation or severe cataract, and age younger than 18 years. In this cross-sectional design study, a total of 166 eyes of 83 subjects aged 18-40 years were enrolled. The participants were divided randomly into 3 groups based on application of drops. Subjects who received a drop of 1\% Tropicamide (anticholinergic blocking muscarinic receptors $\mathrm{M}$ 4) 3 times at 5 min intervals were referred to as the Tropicamide group, while subjects who received a drop of $2.5 \%$ phenylephrine (alpha agonist with sympathomimetic effect) 3 times at $5 \mathrm{~min}$ intervals were the phenylephrine group. Subjects who received a drop of $1 \%$ cyclopentolate (muscarinic antagonist) 3 times at 5 min intervals were defined as the cyclopentolate group. All subjects underwent a full ophthalmic examination involving; 1 . Visual acuity and refraction. 2. Slit- lamp biomicroscopy.3. Fundus examination. 4. IOP measurement using noncontact tonometry. 5. Anterior chamber parameters measurement using Pentacam Rotating Scheimpflug Camera (Pentacam HR - 70900 Oculus).6. Choroidal thickness measurement by the OCT (3D OCT-2000 Spectral Domain OCT).

Procedure of image acquisition by pentacam and OCT has been previously described. [11,12] For each subject, corneal thickness, volume, curvature, anterior chamber depth, volume and angle were acquired through Pentacam before and 50 minutes after instillation of drops.

The sub foveal, temporal, and nasal choroidal thickness were defined as the vertical distance from the hyper reflective line of bruchs membrane to the hyper reflective line of the inner surface of the sclera, each vertical scan is separated by $1 \mathrm{~mm}$ (Figure 1). All subjects were imaged by the same examiner. OCT images of each subject were obtained before the administration of drops and after instillation. All scans were accomplished around the same time of the day, to avoid diurnal choroidal thickness fluctuations.

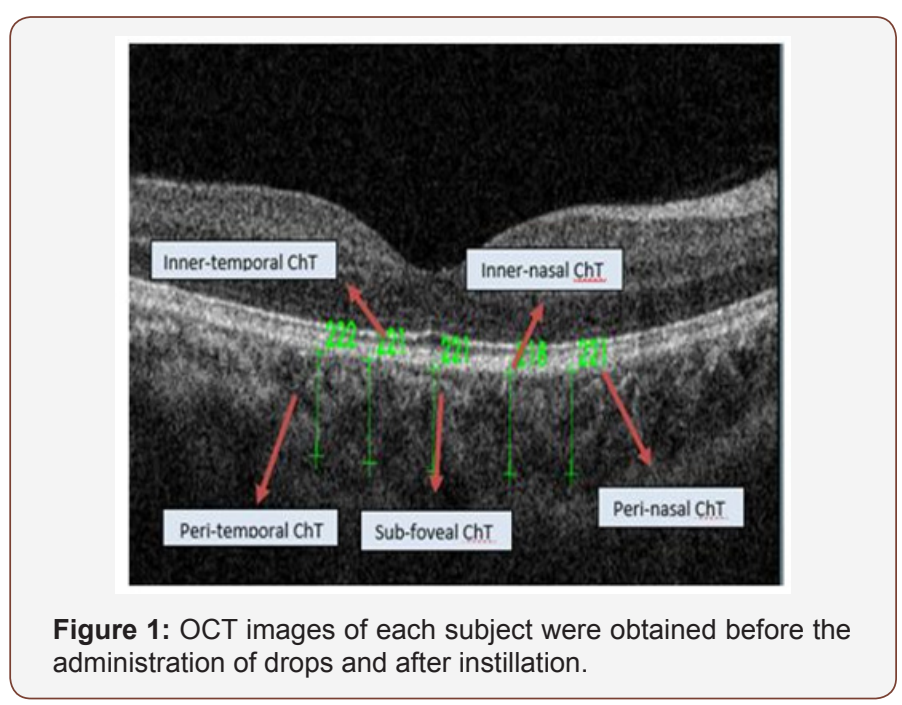

\section{Statistical analysis}

Table 1: Descriptive variables (ChT).

\begin{tabular}{|c|c|c|c|c|c|c|c|c|}
\hline & & \multirow{2}{*}{$\begin{array}{c}\mathbf{N} \\
(\mu \mathrm{m})\end{array}$} & \multirow[t]{2}{*}{ Mean } & \multirow[b]{2}{*}{ Std. Deviation } & \multicolumn{2}{|c|}{$\begin{array}{l}\text { 95\% Confidence Interval } \\
\text { for Mean }\end{array}$} & \multirow[b]{2}{*}{ Minimum } & \multirow[b]{2}{*}{ Maximum } \\
\hline & & & & & $\begin{array}{l}\text { Lower } \\
\text { Bound }\end{array}$ & $\begin{array}{l}\text { Upper } \\
\text { Bound }\end{array}$ & & \\
\hline \multirow{4}{*}{ peri_tem_PRE } & CYCLO & 48 & 218.7292 & 13.58933 & 214.7832 & 222.6751 & 195 & 255 \\
\hline & TROPICA & 58 & 217.2586 & 14.76905 & 213.3753 & 221.1419 & 197 & 255 \\
\hline & PHENYL & 60 & 208.15 & 16.96639 & 203.7671 & 212.5329 & 161 & 258 \\
\hline & Total & 166 & 214.3916 & 15.91903 & 211.952 & 216.8311 & 161 & 258 \\
\hline
\end{tabular}




\begin{tabular}{|c|c|c|c|c|c|c|c|c|}
\hline \multirow{4}{*}{ peri_tem_POST } & CYCLO & 48 & 192.4167 & 16.37309 & 187.6624 & 197.1709 & 164 & 220 \\
\hline & TROPICA & 58 & 193.6379 & 12.66533 & 190.3078 & 196.9681 & 170 & 230 \\
\hline & PHENYL & 60 & 184.45 & 20.50583 & 179.1528 & 189.7472 & 115 & 236 \\
\hline & Total & 166 & 189.9639 & 17.31137 & 187.3109 & 192.6168 & 115 & 236 \\
\hline \multirow{4}{*}{ inner_tem_PRE } & CYCLO & 48 & 220.0625 & 13.79702 & 216.0563 & 224.0687 & 191 & 258 \\
\hline & TROPICA & 58 & 218.8276 & 19.62824 & 213.6666 & 223.9886 & 200 & 310 \\
\hline & PHENYL & 60 & 209.4333 & 15.98343 & 205.3044 & 213.5623 & 167 & 258 \\
\hline & Total & 166 & 215.7892 & 17.37564 & 213.1264 & 218.4519 & 167 & 310 \\
\hline \multirow{4}{*}{ inner_tem_POST } & CYCLO & 48 & 195.3958 & 22.82844 & 188.7671 & 202.0245 & 161 & 303 \\
\hline & TROPICA & 58 & 194.3966 & 12.63376 & 191.0747 & 197.7184 & 172 & 233 \\
\hline & PHENYL & 60 & 185.0333 & 20.765 & 179.6692 & 190.3975 & 118 & 237 \\
\hline & Total & 166 & 191.3012 & 19.50119 & 188.3127 & 194.2897 & 118 & 303 \\
\hline \multirow{4}{*}{ subfoveal_PRE } & CYCLO & 48 & 222.6042 & 21.33396 & 216.4094 & 228.7989 & 191 & 333 \\
\hline & TROPICA & 58 & 216.7241 & 14.91942 & 212.8013 & 220.647 & 195 & 256 \\
\hline & PHENYL & 60 & 209.2333 & 15.74626 & 205.1656 & 213.301 & 168 & 250 \\
\hline & Total & 166 & 215.7169 & 18.01778 & 212.9557 & 218.478 & 168 & 333 \\
\hline \multirow{4}{*}{ subfoveal_POST } & CYCLO & 48 & 195.5417 & 21.45055 & 189.3131 & 201.7703 & 160 & 286 \\
\hline & TROPICA & 58 & 195.7759 & 12.36299 & 192.5252 & 199.0265 & 169 & 227 \\
\hline & PHENYL & 60 & 185.3167 & 21.03467 & 179.8828 & 190.7505 & 115 & 236 \\
\hline & Total & 166 & 191.9277 & 19.15663 & 188.992 & 194.8634 & 115 & 286 \\
\hline \multirow{4}{*}{ inner_nas_PRE } & CYCLO & 48 & 222.0833 & 22.3472 & 215.5944 & 228.5723 & 191 & 334 \\
\hline & TROPICA & 58 & 216.431 & 15.75594 & 212.2882 & 220.5738 & 197 & 258 \\
\hline & PHENYL & 60 & 208.3667 & 14.98809 & 204.4948 & 212.2385 & 170 & 251 \\
\hline & Total & 166 & 215.1506 & 18.4291 & 212.3264 & 217.9748 & 170 & 334 \\
\hline \multirow{4}{*}{ inner_nas_POST } & CYCLO & 48 & 195 & 23.19391 & 188.2652 & 201.7348 & 161 & 305 \\
\hline & TROPICA & 58 & 193.2414 & 12.88316 & 189.8539 & 196.6288 & 162 & 227 \\
\hline & PHENYL & 60 & 184.5167 & 21.53554 & 178.9534 & 190.0799 & 113 & 237 \\
\hline & Total & 166 & 190.5964 & 19.94872 & 187.5393 & 193.6535 & 113 & 305 \\
\hline \multirow{4}{*}{ peri_nas_PRE } & CYCLO & 48 & 219.1667 & 15.18304 & 214.758 & 223.5754 & 190 & 268 \\
\hline & TROPICA & 58 & 217 & 15.06594 & 213.0386 & 220.9614 & 198 & 258 \\
\hline & PHENYL & 60 & 208.5167 & 16.44456 & 204.2686 & 212.7647 & 171 & 255 \\
\hline & Total & 166 & 214.5602 & 16.19631 & 212.0782 & 217.0423 & 171 & 268 \\
\hline \multirow{4}{*}{ peri_nas_POST } & CYCLO & 48 & 193.4375 & 17.00927 & 188.4985 & 198.3765 & 162 & 225 \\
\hline & TROPICA & 58 & 193.0172 & 12.15036 & 189.8225 & 196.212 & 169 & 225 \\
\hline & PHENYL & 60 & 184.6333 & 20.57147 & 179.3192 & 189.9475 & 116 & 236 \\
\hline & Total & 166 & 190.1084 & 17.37327 & 187.446 & 192.7708 & 116 & 236 \\
\hline
\end{tabular}

Table 2: Descriptive variables (Anterior chamber parameters).

\begin{tabular}{|c|c|c|c|c|c|c|c|c|}
\hline & & \multirow{2}{*}{$\mathbf{N}$} & \multirow{2}{*}{ Mean } & \multirow{2}{*}{ Std. Deviation } & \multicolumn{2}{|c|}{$95 \%$ Confidence Interval for Mean } & \multirow{2}{*}{ Minimum } & \multirow{2}{*}{ Maximum } \\
\hline & & & & & Lower Bound & Upper Bound & & \\
\hline \multirow{4}{*}{ CT_PRE $(\mu \mathrm{m})$} & CYCLO & 48 & 557.9792 & 37.58819 & 547.0647 & 568.8936 & 497 & 659 \\
\hline & TROPICA & 58 & 556.7241 & 30.72332 & 548.6459 & 564.8024 & 497 & 628 \\
\hline & PHENYL & 60 & 546.55 & 34.07341 & 537.7479 & 555.3521 & 492 & 645 \\
\hline & Total & 166 & 553.4096 & 34.2159 & 548.1662 & 558.6531 & 492 & 659 \\
\hline \multirow{4}{*}{ CT_POST $(\mu \mathrm{m})$} & CYCLO & 48 & 563.0208 & 36.31745 & 552.4753 & 573.5663 & 504 & 658 \\
\hline & TROPICA & 58 & 556.7241 & 28.34115 & 549.2722 & 564.1761 & 508 & 605 \\
\hline & PHENYL & 60 & 553.2167 & 32.58995 & 544.7978 & 561.6355 & 500 & 654 \\
\hline & Total & 166 & 557.2771 & 32.38305 & 552.3145 & 562.2397 & 500 & 658 \\
\hline
\end{tabular}




\begin{tabular}{|c|c|c|c|c|c|c|c|c|}
\hline \multirow{4}{*}{ CV_PRE $\left(\mathrm{mm}^{3}\right)$} & CYCLO & 48 & 60.4688 & 4.0416 & 59.2952 & 61.6423 & 51.5 & 69.9 \\
\hline & TROPICA & 58 & 62.519 & 13.60656 & 58.9413 & 66.0966 & 54.1 & 161 \\
\hline & PHENYL & 60 & 59.2633 & 3.68151 & 58.3123 & 60.2144 & 51.5 & 69.3 \\
\hline & Total & 166 & 60.7494 & 8.68233 & 59.4189 & 62.0799 & 51.5 & 161 \\
\hline \multirow{4}{*}{$\begin{array}{c}\text { CV_POST } \\
\text { (mm3) }\end{array}$} & CYCLO & 48 & 60.1813 & 4.65017 & 58.831 & 61.5315 & 46 & 69 \\
\hline & TROPICA & 58 & 62.0414 & 13.16794 & 58.579 & 65.5037 & 52.5 & 157 \\
\hline & PHENYL & 60 & 59.48 & 3.6279 & 58.5428 & 60.4172 & 53.4 & 70.7 \\
\hline & Total & 166 & 60.5777 & 8.48545 & 59.2773 & 61.8781 & 46 & 157 \\
\hline \multirow{4}{*}{ CC_PRE (D) } & CYCLO & 48 & 42.7875 & 1.14884 & 42.4539 & 43.1211 & 40.1 & 45.2 \\
\hline & TROPICA & 58 & 42.6586 & 1.74276 & 42.2004 & 43.1169 & 39.7 & 46 \\
\hline & PHENYL & 60 & 43.2433 & 2.3796 & 42.6286 & 43.858 & 40.3 & 59.1 \\
\hline & Total & 166 & 42.9072 & 1.87534 & 42.6198 & 43.1946 & 39.7 & 59.1 \\
\hline \multirow{4}{*}{ CC_POST (D) } & CYCLO & 48 & 42.6021 & 1.91283 & 42.0467 & 43.1575 & 32.7 & 45.4 \\
\hline & TROPICA & 58 & 42.6207 & 1.80582 & 42.1459 & 43.0955 & 38.7 & 45.9 \\
\hline & PHENYL & 60 & 43.015 & 1.1558 & 42.7164 & 43.3136 & 40.3 & 45.3 \\
\hline & Total & 166 & 42.7578 & 1.63834 & 42.5068 & 43.0089 & 32.7 & 45.9 \\
\hline \multirow{4}{*}{ ACD_PRE (mm) } & CYCLO & 48 & 3.1102 & 0.256 & 3.0359 & 3.1845 & 2.5 & 3.74 \\
\hline & TROPICA & 58 & 3.079 & 0.28814 & 3.0032 & 3.1547 & 2.37 & 3.65 \\
\hline & PHENYL & 60 & 6.1028 & 23.61932 & 0.0013 & 12.2044 & 2.52 & 186 \\
\hline & Total & 166 & 4.181 & 14.19973 & 2.0049 & 6.357 & 2.37 & 186 \\
\hline \multirow{4}{*}{$\begin{array}{c}\text { ACD_POST } \\
(\mathrm{mm})\end{array}$} & CYCLO & 48 & 3.1671 & 0.25283 & 3.0937 & 3.2405 & 2.5 & 3.84 \\
\hline & TROPICA & 58 & 3.164 & 0.25606 & 3.0966 & 3.2313 & 2.64 & 3.8 \\
\hline & PHENYL & 60 & 3.0947 & 0.23134 & 3.0349 & 3.1544 & 2.52 & 3.65 \\
\hline & Total & 166 & 3.1398 & 0.2473 & 3.1019 & 3.1777 & 2.5 & 3.84 \\
\hline \multirow{4}{*}{$\begin{array}{c}\text { ACV_PRE } \\
(\mathrm{mm} 3)\end{array}$} & CYCLO & 48 & 181.8125 & 33.13394 & 172.1914 & 191.4336 & 125 & 269 \\
\hline & TROPICA & 58 & 173.0345 & 28.53312 & 165.5321 & 180.5369 & 112 & 244 \\
\hline & PHENYL & 60 & 175.0345 & 38.46369 & 165.0983 & 184.9707 & 3.07 & 308 \\
\hline & Total & 166 & 176.2956 & 33.70696 & 171.1301 & 181.4611 & 3.07 & 308 \\
\hline \multirow{4}{*}{$\begin{array}{c}\text { ACV_POST } \\
\text { (mm3) }\end{array}$} & CYCLO & 48 & 189.4167 & 33.6065 & 179.6584 & 199.175 & 107 & 267 \\
\hline & TROPICA & 58 & 185.569 & 30.31652 & 177.5976 & 193.5403 & 112 & 275 \\
\hline & PHENYL & 60 & 184.65 & 27.00836 & 177.673 & 191.627 & 125 & 247 \\
\hline & Total & 166 & 186.3494 & 30.06737 & 181.7417 & 190.9571 & 107 & 275 \\
\hline \multirow{4}{*}{ ACA_PRE $\left({ }^{\circ}\right)$} & CYCLO & 48 & 38.5104 & 4.23156 & 37.2817 & 39.7391 & 26.8 & 47.9 \\
\hline & TROPICA & 58 & 38.3017 & 3.93212 & 37.2678 & 39.3356 & 30.9 & 47.1 \\
\hline & PHENYL & 60 & 39.3767 & 3.96341 & 38.3528 & 40.4005 & 26.7 & 49.9 \\
\hline & Total & 166 & 38.7506 & 4.03596 & 38.1321 & 39.3691 & 26.7 & 49.9 \\
\hline \multirow{4}{*}{ ACA_POST $\left({ }^{\circ}\right)$} & CYCLO & 48 & 38.8604 & 6.50449 & 36.9717 & 40.7491 & 25.9 & 53.4 \\
\hline & TROPICA & 58 & 46.2431 & 40.07739 & 35.7053 & 56.7809 & 20.4 & 196 \\
\hline & PHENYL & 60 & 39.15 & 5.25007 & 37.7938 & 40.5062 & 26 & 49 \\
\hline & Total & 166 & 41.5446 & 24.26349 & 37.8263 & 45.2629 & 20.4 & 196 \\
\hline
\end{tabular}

All data analyses were performed by using the Statistical Package for the Social Sciences (SPSS) for Windows version 20.0. All variables were presented as mean \pm standard deviation (Table 1-2). A Paired $t$-test was done to compare the measurements of anterior chamber parameters and choroidal thickness (ChT) of the Tropicamide group (Table 3), Phenylephrine group (Table 4) and
Cyclopentolate group (Table 5). The Homogeneity of variances was confirmed by using Levene's test. For parametric statistics, the data were normally distributed and were analyzed using one-way ANOVA test (Table 6) to compare the groups. When a significant result was obtained, the Tukey test was used for Post hoc comparisons (Table 7). A $p$ value $<0.05$ was considered statistically significant. 
Table 3: Paired $t$-test (Tropicamide group).

\begin{tabular}{|c|c|c|c|c|c|c|c|c|}
\hline & & \multirow[t]{2}{*}{ Mean } & \multirow[t]{2}{*}{ Std. Deviation } & \multicolumn{2}{|c|}{$\begin{array}{l}\text { 95\% Confidence Interval of the } \\
\text { Difference }\end{array}$} & \multirow[t]{2}{*}{$\mathbf{t}$} & \multirow[t]{2}{*}{ df } & \multirow[t]{2}{*}{ Sig. (2-tailed) } \\
\hline & & & & Lower & Upper & & & \\
\hline Pair 1 & CT_PRE - POST & 0 & 17.89639 & -4.70562 & 4.70562 & 0 & 57 & 1 \\
\hline Pair 2 & CV_PRE - POST & 0.47759 & 18.96852 & -4.50993 & 5.4651 & 0.192 & 57 & 0.849 \\
\hline Pair 3 & CC_PRE - POST & 0.03793 & 0.24909 & -0.02756 & 0.10343 & 1.16 & 57 & 0.251 \\
\hline Pair 4 & ACD_PRE - POST & -0.085 & 0.08146 & -0.10642 & -0.06358 & -7.947 & 57 & $.000^{* *}$ \\
\hline Pair 5 & ACV_PRE - POST & -12.53448 & 16.57436 & -16.89249 & -8.17648 & -5.759 & 57 & $.000^{* *}$ \\
\hline Pair 6 & ACA_PRE - POST & -7.94138 & 39.70284 & -18.38071 & 2.49795 & -1.523 & 57 & 0.133 \\
\hline Pair 7 & peri_tem_PRE- POST & 23.62069 & 19.34709 & 18.53363 & 28.70775 & 9.298 & 57 & $.000^{* *}$ \\
\hline Pair 8 & inner_tem_PRE- POST & 24.43103 & 22.58171 & 18.49348 & 30.36859 & 8.239 & 57 & $.000^{* *}$ \\
\hline Pair 9 & Sub foveal_PRE-POST & 20.94828 & 19.27677 & 15.87971 & 26.01684 & 8.276 & 57 & $.000^{* *}$ \\
\hline Pair 10 & Inner_nas_PRE- POST & 23.18966 & 20.22458 & 17.87187 & 28.50744 & 8.732 & 57 & $.000^{* *}$ \\
\hline Pair 11 & peri_nas_PRE- POST & 23.98276 & 19.50753 & 18.85352 & 29.112 & 9.363 & 57 & $.000^{* *}$ \\
\hline
\end{tabular}

* Significant. * *. Highly significant.

Table 4: Paired t-test (Phenylephrine group).

\begin{tabular}{|c|c|c|c|c|c|c|c|c|}
\hline & & \multirow[t]{2}{*}{ Mean } & \multirow[t]{2}{*}{ Std. Deviation } & \multicolumn{2}{|c|}{$\begin{array}{l}\text { 95\% Confidence Interval of the } \\
\text { Difference }\end{array}$} & \multirow[t]{2}{*}{$\mathrm{t}$} & \multirow[t]{2}{*}{ df } & \multirow[t]{2}{*}{ Sig. (2-tailed) } \\
\hline & & & & Lower & Upper & & & \\
\hline Pair 1 & CT_PRE - POST & -5.81395 & 16.92175 & -11.0217 & -0.60621 & -2.253 & 42 & $.030^{*}$ \\
\hline Pair 2 & CV_PRE - POST & -0.21628 & 0.94565 & -0.50731 & 0.07475 & -1.5 & 42 & 0.141 \\
\hline Pair 3 & CC_PRE - POST & 0.35116 & 2.57195 & -0.44037 & 1.14269 & 0.895 & 42 & 0.376 \\
\hline Pair 4 & ACD_PRE -POST & 4.22047 & 27.90503 & -4.36744 & 12.80837 & 0.992 & 42 & 0.327 \\
\hline Pair 5 & ACV_PRE -POST & -11.81233 & 26.15752 & -19.86242 & -3.76223 & -2.961 & 42 & $.005^{* *}$ \\
\hline Pair 6 & ACA_PRE - POST & 0.80465 & 4.94055 & -0.71583 & 2.32513 & 1.068 & 42 & 0.292 \\
\hline Pair 7 & peri_tem_PRE -POST & 24.32558 & 14.912 & 19.73635 & 28.91482 & 10.697 & 42 & $.000^{* *}$ \\
\hline Pair 8 & inner_tem_PRE- POST & 25.97674 & 13.80043 & 21.7296 & 30.22389 & 12.343 & 42 & $.000^{* *}$ \\
\hline Pair 9 & subfoveal_PRE -POST & 25.2093 & 14.2621 & 20.82008 & 29.59853 & 11.591 & 42 & $.000^{* *}$ \\
\hline Pair 10 & inner_nas_PRE -POST & 24.83721 & 16.18925 & 19.85489 & 29.81953 & 10.06 & 42 & $.000^{* *}$ \\
\hline Pair 11 & peri_nas_PRE -POST & 24.76744 & 16.4575 & 19.70257 & 29.83231 & 9.869 & 42 & $.000^{* *}$ \\
\hline
\end{tabular}

Table 5: Paired t-test (Cyclopentolate group).

\begin{tabular}{|c|c|c|c|c|c|c|c|c|}
\hline & & Mean & Std. Deviation & 95\% Confic & rval of the Dif- & $\mathbf{t}$ & df & Sig. (2-tailed) \\
\hline & & & & Lower & Upper & & & \\
\hline Pair 1 & CT_PRE - POST & -5.04167 & 15.44098 & -9.52526 & -0.55807 & -2.262 & 47 & $.028^{*}$ \\
\hline Pair 2 & CV_PRE - POST & 0.2875 & 2.45293 & -0.42476 & 0.99976 & 0.812 & 47 & 0.421 \\
\hline Pair 3 & CC_PRE - POST & 0.18542 & 1.47979 & -0.24427 & 0.6151 & 0.868 & 47 & 0.39 \\
\hline Pair 4 & ACD_PRE - POST & -0.05688 & 0.14569 & -0.09918 & -0.01457 & -2.705 & 47 & $.009 * *$ \\
\hline Pair 5 & ACV_PRE - POST & -7.60417 & 17.84417 & -12.78557 & -2.42276 & -2.952 & 47 & $.005^{* *}$ \\
\hline Pair 6 & ACA_PRE - POST & -0.35 & 6.10967 & -2.12406 & 1.42406 & -0.397 & 47 & 0.693 \\
\hline Pair 7 & peri_tem_PRE- POST & 26.3125 & 18.79357 & 20.85542 & 31.76958 & 9.7 & 47 & $.000 * *$ \\
\hline Pair 8 & inner_tem_PRE- POST & 24.66667 & 25.09754 & 17.3791 & 31.95423 & 6.809 & 47 & $.000^{* *}$ \\
\hline Pair 9 & subfoveal_PRE-POST & 27.0625 & 27.64933 & 19.03397 & 35.09103 & 6.781 & 47 & $.000^{* *}$ \\
\hline Pair 10 & inner_nas_PRE-POST & 27.08333 & 20.56627 & 21.11151 & 33.05516 & 9.124 & 47 & $.000^{* *}$ \\
\hline Pair 11 & peri_nas_PRE -POST & 25.72917 & 20.53408 & 19.76669 & 31.69164 & 8.681 & 47 & $.000^{* *}$ \\
\hline
\end{tabular}


Table 6: One-way ANOVA test.

\begin{tabular}{|c|c|c|c|c|c|}
\hline & & Sum of Squares & df & Mean Square & Sig. \\
\hline \multirow{3}{*}{ peri_tem_PRE } & Between Groups & 3717.298 & 2 & 1858.649 & $.001^{* *}$ \\
\hline & Within Groups & 38096.25 & 163 & 233.719 & \\
\hline & Total & 41813.548 & 165 & & \\
\hline \multirow{3}{*}{ peri_tem_POST } & Between Groups & 2895.87 & 2 & 1447.935 & $.007^{* *}$ \\
\hline & Within Groups & 46551.913 & 163 & 285.595 & \\
\hline & Total & 49447.783 & 165 & & \\
\hline \multirow{3}{*}{ inner_tem_PRE } & Between Groups & 3835.799 & 2 & 1917.899 & $.001^{* *}$ \\
\hline & Within Groups & 45979.822 & 163 & 282.085 & \\
\hline & Total & 49815.62 & 165 & & \\
\hline \multirow{3}{*}{ innet_tem_POST } & Between Groups & 3717.648 & 2 & 1858.824 & $.007^{* *}$ \\
\hline & Within Groups & 59031.292 & 163 & 362.155 & \\
\hline & Total & 62748.94 & 165 & & \\
\hline \multirow{3}{*}{ subfoveal_PRE } & Between Groups & 4857.894 & 2 & 2428.947 & $.000^{* *}$ \\
\hline & Within Groups & 48707.799 & 163 & 298.821 & \\
\hline & Total & 53565.693 & 165 & & \\
\hline \multirow{3}{*}{ subfoveal_POST } & Between Groups & 4108.146 & 2 & 2054.073 & $.003^{* *}$ \\
\hline & Within Groups & 56442.986 & 163 & 346.276 & \\
\hline & Total & 60551.133 & 165 & & \\
\hline \multirow{3}{*}{ inner_nas_PRE } & Between Groups & 5163.411 & 2 & 2581.705 & $.000^{* *}$ \\
\hline & Within Groups & 50875.824 & 163 & 312.122 & \\
\hline & Total & 56039.235 & 165 & & \\
\hline \multirow{3}{*}{ inner_nas_POST } & Between Groups & 3554.354 & 2 & 1777.177 & $.011^{* *}$ \\
\hline & Within Groups & 62107.604 & 163 & 381.028 & \\
\hline & Total & 65661.958 & 165 & & \\
\hline \multirow{3}{*}{ peri_nas_PRE } & Between Groups & 3555.248 & 2 & 1777.624 & $.001^{* *}$ \\
\hline & Within Groups & 39727.65 & 163 & 243.728 & \\
\hline & Total & 43282.898 & 165 & & \\
\hline \multirow{3}{*}{ peri_nas_POST } & Between Groups & 2821.32 & 2 & 1410.66 & $.009^{* *}$ \\
\hline & Within Groups & 46980.729 & 163 & 288.225 & \\
\hline & Total & 49802.048 & 165 & & \\
\hline
\end{tabular}

Table 7: Post Hoc Test.

\begin{tabular}{|c|c|c|c|c|c|c|}
\hline \multirow{2}{*}{ Dependent Variable } & \multirow{2}{*}{ (I) Groups } & \multirow{2}{*}{ (J) Groups } & \multirow{2}{*}{ Mean Difference (I-J) } & \multirow{2}{*}{ Sig. } & \multicolumn{2}{|c|}{ 95\% Confidence Interval } \\
\hline & & & & & Lower Bound & Upper Bound \\
\hline \multirow{6}{*}{ peri_tem_PRE } & \multirow{2}{*}{ CYCLO } & TROPICA & 1.47055 & 0.875 & -5.5854 & 8.5265 \\
\hline & & PHENYL & $10.57917^{*}$ & $.001^{* *}$ & 3.5767 & 17.5817 \\
\hline & \multirow{2}{*}{ TROPICA } & CYCLO & -1.47055 & 0.875 & -8.5265 & 5.5854 \\
\hline & & PHENYL & $9.10862^{*}$ & $.004^{* *}$ & 2.4499 & 15.7673 \\
\hline & \multirow{2}{*}{ PHENYL } & CYCLO & $-10.57917^{*}$ & $.001^{* *}$ & -17.5817 & -3.5767 \\
\hline & & TROPICA & $-9.10862 *$ & $.004^{* *}$ & -15.7673 & -2.4499 \\
\hline \multirow{6}{*}{ peri_tem_POST } & \multirow{2}{*}{ CYCLO } & TROPICA & -1.22126 & 0.927 & -9.0211 & 6.5785 \\
\hline & & PHENYL & $7.96667^{*}$ & $.042^{* *}$ & 0.226 & 15.7074 \\
\hline & \multirow{2}{*}{ TROPICA } & CYCLO & 1.22126 & 0.927 & -6.5785 & 9.0211 \\
\hline & & PHENYL & $9.18793^{*}$ & $.010^{* *}$ & 1.8273 & 16.5486 \\
\hline & \multirow{2}{*}{ PHENYL } & CYCLO & $-7.96667^{*}$ & $.042^{*}$ & -15.7074 & -0.226 \\
\hline & & TROPICA & $-9.18793^{*}$ & $.010^{* *}$ & -16.5486 & -1.8273 \\
\hline
\end{tabular}




\begin{tabular}{|c|c|c|c|c|c|c|}
\hline \multirow{6}{*}{ inner_tem_PRE } & \multirow{2}{*}{ CYCLO } & TROPICA & 1.23491 & 0.925 & -6.5168 & 8.9866 \\
\hline & & PHENYL & $10.62917^{*}$ & $.004^{* *}$ & 2.9362 & 18.3222 \\
\hline & \multirow{2}{*}{ TROPICA } & CYCLO & -1.23491 & 0.925 & -8.9866 & 6.5168 \\
\hline & & PHENYL & $9.39425^{*}$ & $.008^{* *}$ & 2.079 & 16.7095 \\
\hline & \multirow{2}{*}{ PHENYL } & CYCLO & $-10.62917^{*}$ & $.004^{* *}$ & -18.3222 & -2.9362 \\
\hline & & TROPICA & $-9.39425^{*}$ & $.008^{* *}$ & -16.7095 & -2.079 \\
\hline \multirow{6}{*}{ innet_tem_POST } & \multirow{2}{*}{ CYCLO } & TROPICA & 0.99928 & 0.961 & -7.784 & 9.7825 \\
\hline & & PHENYL & $10.36250^{*}$ & $.015^{* *}$ & 1.6458 & 19.0792 \\
\hline & \multirow{2}{*}{ TROPICA } & CYCLO & -0.99928 & 0.961 & -9.7825 & 7.784 \\
\hline & & PHENYL & $9.36322^{*}$ & $.023^{* *}$ & 1.0745 & 17.652 \\
\hline & \multirow{2}{*}{ PHENYL } & CYCLO & $-10.36250^{*}$ & $.015^{* *}$ & -19.0792 & -1.6458 \\
\hline & & TROPICA & $-9.36322^{*}$ & $.023^{* *}$ & -17.652 & -1.0745 \\
\hline \multirow{6}{*}{ subfoveal_PRE } & \multirow{2}{*}{ CYCLO } & TROPICA & 5.88003 & 0.192 & -2.0983 & 13.8584 \\
\hline & & PHENYL & 13.37083* & $.000^{* *}$ & 5.4529 & 21.2888 \\
\hline & \multirow{2}{*}{ TROPICA } & CYCLO & -5.88003 & 0.192 & -13.8584 & 2.0983 \\
\hline & & PHENYL & 7.4908 & 0.052 & -0.0384 & 15.02 \\
\hline & \multirow{2}{*}{ PHENYL } & CYCLO & $-13.37083^{*}$ & $.000 * *$ & -21.2888 & -5.4529 \\
\hline & & TROPICA & -7.4908 & 0.052 & -15.02 & 0.0384 \\
\hline \multirow{6}{*}{ subfoveal_POST } & \multirow{2}{*}{ CYCLO } & TROPICA & -0.2342 & 0.998 & -8.8227 & 8.3543 \\
\hline & & PHENYL & $10.22500^{*}$ & $.014^{* *}$ & 1.7015 & 18.7485 \\
\hline & \multirow{2}{*}{ TROPICA } & CYCLO & 0.2342 & 0.998 & -8.3543 & 8.8227 \\
\hline & & PHENYL & $10.45920^{*}$ & $.007^{* *}$ & 2.3542 & 18.5642 \\
\hline & \multirow{2}{*}{ PHENYL } & CYCLO & $-10.22500^{*}$ & $.014^{* *}$ & -18.7485 & -1.7015 \\
\hline & & TROPICA & $-10.45920^{*}$ & $.007^{* *}$ & -18.5642 & -2.3542 \\
\hline \multirow{6}{*}{ inner_nas_PRE } & \multirow{2}{*}{ CYCLO } & TROPICA & 5.6523 & 0.232 & -2.5017 & 13.8063 \\
\hline & & PHENYL & $13.71667 *$ & $.000^{* *}$ & 5.6245 & 21.8089 \\
\hline & \multirow{2}{*}{ TROPICA } & CYCLO & -5.6523 & 0.232 & -13.8063 & 2.5017 \\
\hline & & PHENYL & $8.06437^{*}$ & $.038^{*}$ & 0.3695 & 15.7593 \\
\hline & \multirow{2}{*}{ PHENYL } & CYCLO & $-13.71667^{*}$ & $.000^{* *}$ & -21.8089 & -5.6245 \\
\hline & & TROPICA & $-8.06437^{*}$ & $.038^{*}$ & -15.7593 & -0.3695 \\
\hline \multirow{6}{*}{ inner_nas_POST } & \multirow{2}{*}{ CYCLO } & TROPICA & 1.75862 & 0.889 & -7.2506 & 10.7678 \\
\hline & & PHENYL & $10.48333^{*}$ & $.017^{* *}$ & 1.5424 & 19.4243 \\
\hline & \multirow{2}{*}{ TROPICA } & CYCLO & -1.75862 & 0.889 & -10.7678 & 7.2506 \\
\hline & & PHENYL & $8.72471^{*}$ & $.043^{*}$ & 0.2227 & 17.2267 \\
\hline & \multirow{2}{*}{ PHENYL } & CYCLO & $-10.48333^{*}$ & $.017^{* *}$ & -19.4243 & -1.5424 \\
\hline & & TROPICA & $-8.72471^{*}$ & $.043^{*}$ & -17.2267 & -0.2227 \\
\hline 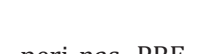 & CУCIO & TROPICA & 2.16667 & 0.757 & -5.0388 & 9.3721 \\
\hline 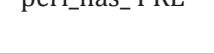 & CicLo & PHENYL & $10.65000^{*}$ & $.002^{* *}$ & 3.4991 & 17.8009 \\
\hline & TROPICA & CYCLO & -2.16667 & 0.757 & -9.3721 & 5.0388 \\
\hline & Н & PHENYL & 8.48333* & $.010^{* *}$ & 1.6836 & 15.2831 \\
\hline & DHFNYI & CYCLO & $-10.65000^{*}$ & $.002^{* *}$ & -17.8009 & -3.4991 \\
\hline & PHENYL & TROPICA & $-8.48333^{*}$ & $.010^{* *}$ & -15.2831 & -1.6836 \\
\hline nori nas POCT & CYCLO & TROPICA & 0.42026 & 0.991 & -7.4154 & 8.2559 \\
\hline pei1_nas_t & GicLo & PHENYL & $8.80417^{*}$ & $.022^{* *}$ & 1.0279 & 16.5804 \\
\hline
\end{tabular}




\begin{tabular}{|l|c|c|c|c|c|c|}
\hline \multirow{2}{*}{ TROPICA } & CYCLO & -0.42026 & 0.991 & -8.2559 & 7.4154 \\
\cline { 3 - 7 } & \multirow{2}{*}{ PHENYL } & PHENYL & $8.38391^{*}$ & $\mathbf{. 0 2 2}^{* *}$ & 0.9894 & 15.7784 \\
\hline & & CYCLO & $-8.80417^{*}$ & $\mathbf{. 0 2 2}^{* *}$ & -16.5804 & -1.0279 \\
\cline { 3 - 7 } & & TROPICA & $-8.38391^{*}$ & $\mathbf{. 0 2 2}^{* *}$ & -15.7784 & -0.9894 \\
\hline
\end{tabular}

\section{Result}

\section{Demographic data}

The mean age was 21.6 \pm 1.6 years (age range:18-23 years). The subjects were divided randomly into 3 groups based on the application of the drops. Groups were comprised of tropicamide group $(n=58 ; 34.7 \%)$, the phenylephrine group $(n=60 ; 35.9 \%)$, and cyclopentolate group $(n=48 ; 28.7 \%)$. The mean spherical equivalent was measured as $-1.37 \pm 1.73$ diopters in the tropicamide group, $-1.46 \pm 1.83$ diopters in the phenylephrine group, and $-1.62 \pm 1.63$ diopters in the cyclopentolate group. There were no significant differences in the demographic characteristics and ocular parameters between the 3 groups. Demographics data in all groups were summarized in (Table 8), including the mean age, best corrected visual acuity, and the spherical equivalent power.

Table 8: Demographic data.

\begin{tabular}{|c|c|c|c|}
\hline Cyclopentolate & Phenylephrine & Tropicamide & \multirow{2}{*}{} \\
\cline { 1 - 3 } $\mathbf{n = 4 8}$ & $\mathbf{n}=\mathbf{6 0}$ & $\mathbf{n}=\mathbf{5 8}$ & \\
\hline $21.8 \pm 2.2$ & $21.4 \pm 1.1$ & $21.8 \pm 1.6$ & Age (year) \\
\hline 0 & 0 & 0 & BCVA (log Mar) \\
\hline$-1.62 \pm 1.63$ & $-1.46 \pm 1.83$ & $-1.37 \pm 1.73$ & SEQ (diopters) \\
\hline
\end{tabular}

BCVA: Best Corrected Visual Acuity (0.00 in log Mar $=$ Snellen score of $6 / 6=(20 / 20))$; SEQ: mean spherical equivalent. Variables are expressed as mean \pm standard deviation.

\section{Anterior chamber parameters}

The Corneal thickness in tropicamide group showed no significant difference from $556.72 \pm 30.72 \mu \mathrm{m}$ at baseline to $556.72 \pm 28.34 \mu \mathrm{m}$ after drug administration, corneal thickness increased from $546.55 \pm 30.07 \mu \mathrm{m}$ to $553.21 \pm 32.58 \mu \mathrm{m}$ in the phenylephrine group and increased from $557,97 \pm 37.58 \mu \mathrm{m}$ to $563.02 \pm 36.31 \mu \mathrm{m}$ in the cyclopentolate group. Moreover, the corneal volume in tropicamide group showed no significant change from $62.51 \pm 13.6 \mathrm{~mm} 3$ at baseline to $62.04 \pm 13.16 \mathrm{~mm} 3$ after drug administration, from $59.26 \pm 3.68 \mathrm{~mm} 3$ to $59.48 \pm 3.62 \mathrm{~mm}$ 3in the phenylephrine group, and from $60.46 \pm 4.04 \mathrm{~mm} 3$ to $60.18 \pm 4.65$ $\mathrm{mm} 3$ in the cyclopentolate group.

When it comes to the corneal curvature in tropicamide group it showed no significant change from $42.65 \pm 1.74$ Diopters (D) at baseline to $42.62 \pm 1.91 \mathrm{D}$ after drug administration, from $43.24 \pm$ $2.37 \mathrm{D}$ to $43.01 \pm 1.15 \mathrm{D}$ in the phenylephrine group, and from 42.78 $\pm 1.14 \mathrm{D}$ to $42.60 \pm 1.91 \mathrm{D}$ in the cyclopentolate group.

The results showed that there are no significant changes in the corneal thickness, volume, and curvature after drops instillation in the 3 groups.

The anterior chamber depth showed no significant changes from $3.07 \pm 0.28 \mathrm{~mm}$ at baseline to $3.16 \pm 0.25 \mathrm{~mm}$ after drug administration in the tropicamide group. Anterior chamber depth decreased from $6.10 \pm 23.61 \mathrm{~mm}$ to $3.09 \pm 0.23 \mathrm{~mm}$ in the phenylephrine group. However, it showed no changes from $3.11 \pm$ $0.25 \mathrm{~mm}$ to $3.16 \pm 0.25 \mathrm{~mm}$ in the cyclopentolate group.

However, the anterior chamber volume increased from $173.03 \pm 28.35 \mathrm{~mm}^{3}$ at baseline to $185.56 \pm 30.31 \mathrm{~mm}^{3}$ after drops administration in tropicamide group, from $175.03 \pm 38.46$ $\mathrm{mm}^{3}$ to $184.65 \pm 27 \mathrm{~mm}^{3}$ in the phenylephrine group, and from $181.81 \pm 33.13 \mathrm{~mm}^{3}$ to $189.41 \pm 33.6 \mathrm{~mm}^{3}$ in the cyclopentolate group, respectively. The results showed that the ACV increased significantly in the all groups $(\mathrm{p}=0.000)$.

Furthermore, the angle of the anterior chamber in tropicamide group increased from $38.30 \pm 3.93^{\circ}$ at baseline to $46.24 \pm 40.07^{\circ}$ after drug administrations, the angle of the anterior chamber showed no significant change from $39.37 \pm 3.96^{\circ}$ to $39.15 \pm 5.25^{\circ}$ in the phenylephrine group, and from $38.51 \pm 4.23^{\circ}$ to $38.86 \pm 6.50^{\circ}$ in the cyclopentolate group.

\section{Choroidal thickness}

Concerning the peri-temporal choroidal thickness, we found that there was a significant choroidal thinning in all groups, $(\mathrm{p}=$ 0.00 ). The measurement before and after drops instillation were $217.25 \pm 14.76 \mu$ and $193.63 \pm 12.66 \mu$ in the tropicamide group, $208.15 \pm 16.96 \mu$ and $184.65 \pm 27 \mu$ in the phenylephrine group, $218.72 \pm 13.58 \mu$ and $192.41 \pm 16.37 \mu$ in the cyclopentolate group, respectively.

The subfoveal choroidal thickness measurement before and after drops instillation were, $216.72 \pm 14.91 \mu$ and $195.77 \pm 36 \mu$ in the tropicamide group, $209.23 \pm 15.74 \mu$ and $185.31 \pm 21.03 \mu$ in the phenylephrine group, $222.60 \pm 21.33 \mu$ and $195.54 \pm 21.45 \mu$ in the cyclopentolate group, respectively. These results indicate that the subfoveal choroidal thickness decrease significantly in all groups, $(\mathrm{p}=0.00)$.

Moreover, the peri-nasal choroidal thickness measurement demonstrated a significant reduction in the thickness before and after drops instillation in all groups, $(p=0.00)$. Changes in the anterior chamber parameters and choroidal thickness before and after drops instillation in all groups are demonstrated in (Table 9).

Table 9: Changes of anterior chamber parameters and choroidal thickness before and after drops instillation.

\begin{tabular}{|c|c|c|c|}
\hline Cyclopentolate & Phenylephrine & Tropicamide & \multirow{2}{*}{} \\
\cline { 1 - 3 } $\mathbf{n = 4 8}$ & $\mathbf{n}=\mathbf{6 0}$ & $\mathbf{n}=\mathbf{5 8}$ & \\
\hline $557.97 \pm 37.58$ & $557.97 \pm 37.58$ & $557.97 \pm 37.58$ & CT $(\mu \mathrm{m})$ \\
\hline $563.02 \pm 36.31$ & $563.02 \pm 36.31$ & $563.02 \pm 36.31$ & Before \\
\hline 0.28 & 0.28 & 0.28 & After \\
\hline & & & P value \\
\hline & & & CV $\left(\mathrm{mm}^{3}\right)$ \\
\hline
\end{tabular}




\begin{tabular}{|c|c|c|c|}
\hline $60.46 \pm 4.04$ & $60.46 \pm 4.04$ & $60.46 \pm 4.04$ & Before \\
\hline $60.18 \pm 4.65$ & $60.18 \pm 4.65$ & $60.18 \pm 4.65$ & After \\
\hline \multirow[t]{2}{*}{0.42} & 0.42 & 0.42 & Pvalue \\
\hline & & & CC (D) \\
\hline $42.78 \pm 1.14$ & $42.78 \pm 1.14$ & $42.78 \pm 1.14$ & Before \\
\hline $42.6 \pm 1.91$ & $42.6 \pm 1.91$ & $42.6 \pm 1.91$ & After \\
\hline \multirow[t]{2}{*}{0.39} & 0.39 & 0.39 & $P$ value \\
\hline & & & $\operatorname{ACV}\left(\mathrm{mm}^{3}\right)$ \\
\hline $173.03 \pm 28.53$ & $173.03 \pm 28.53$ & $173.03 \pm 28.53$ & Before \\
\hline $185.56 \pm 30.31$ & $185.56 \pm 30.31$ & $185.56 \pm 30.31$ & After \\
\hline \multirow[t]{2}{*}{0} & 0 & 0 & $P$ value \\
\hline & & & $\mathrm{ACD}(\mathrm{mm})$ \\
\hline $3.11 \pm 0.25$ & $6.1 \pm 23.61$ & $3.07 \pm 0.28$ & Before \\
\hline $3.16 \pm 0.25$ & $3.09 \pm 0.23$ & $3.16 \pm 0.25$ & After \\
\hline \multirow[t]{2}{*}{0.009} & 0.009 & 0.009 & $P$ value \\
\hline & & & $\operatorname{ACA}\left({ }^{\circ}\right)$ \\
\hline $38.51 \pm 4.23$ & $38.51 \pm 4.23$ & $38.51 \pm 4.23$ & Before \\
\hline $38.86 \pm 6.5$ & $38.86 \pm 6.5$ & $38.86 \pm 6.5$ & After \\
\hline \multirow[t]{2}{*}{0.69} & 0.69 & 0.69 & $P$ value \\
\hline & & & $\begin{array}{l}\text { ChT peri-tem- } \\
\text { poral }(\boldsymbol{\mu m})\end{array}$ \\
\hline $218.72 \pm 13.58$ & $208.15 \pm 16.96$ & $217.25 \pm 14.76$ & Before \\
\hline $192.41 \pm 16.37$ & $184.45 \pm 20.5$ & $196.36 \pm 12.66$ & After \\
\hline \multirow[t]{2}{*}{0} & 0 & 0 & $P$ value \\
\hline & & & $\begin{array}{l}\text { ChT inner-tem- } \\
\text { poral }(\mu \mathrm{m})\end{array}$ \\
\hline $220.06 \pm 13.79$ & $209.43 \pm 15.98$ & $218.82 \pm 19.62$ & Before \\
\hline $195.39 \pm 22.82$ & $185.03 \pm 20.76$ & $194.39 \pm 12.63$ & After \\
\hline \multirow[t]{2}{*}{0} & 0 & 0 & $P$ value \\
\hline & & & $\begin{array}{c}\text { ChT subfoveal } \\
(\mu \mathrm{m})\end{array}$ \\
\hline $222.6 \pm 21.33$ & $209.23 \pm 15.74$ & $216.72 \pm 14.91$ & Before \\
\hline $195.54 \pm 21.45$ & $185.31 \pm 21.03$ & $195.77 \pm 12.36$ & After \\
\hline \multirow[t]{2}{*}{0} & 0 & 0 & $P$ value \\
\hline & & & $\begin{array}{l}\text { ChT inner-na- } \\
\text { sal }(\mu \mathrm{m})\end{array}$ \\
\hline $222.08 \pm 22.34$ & $208.36 \pm 14.98$ & $216.43 \pm 15.75$ & Before \\
\hline $195 \pm 23.19$ & $184.51 \pm 21.53$ & $193.24 \pm 12.88$ & After \\
\hline \multirow[t]{2}{*}{0} & 0 & 0 & $P$ value \\
\hline & & & $\begin{array}{c}\text { ChT peri-nasal } \\
(\mu \mathrm{m})\end{array}$ \\
\hline $219.16 \pm 15.18$ & $238.38 \pm 23.06$ & $217 \pm 15.06$ & Before \\
\hline $193.43 \pm 17$ & $184.63 \pm 20.57$ & $193.01 \pm 12.15$ & After \\
\hline 0 & 0 & 0 & $P$ value \\
\hline
\end{tabular}

CT: corneal thickness, CV: corneal volume, CC: corneal curvature, ACV: anterior chamber volume, ACD: anterior chamber depth, ACA: anterior chamber angle, and ChT: choroidal thickness. Variables are expressed as mean \pm standard deviation. Level of significance $p<0.05$, a paired t-test.

\section{Discussion}

Current study carried out on 166 eyes of 83 subjects; found that all mydriatic agents led to significant choroidal thinning. It showed that there were changes at the level of the anterior chamber parameters. Especially ACV that increased significantly in all groups.

N Kara et al. [10] conducted a study on 90 healthy subjects to investigate the effect of 2 commonly used mydriatics (tropicamide and phenylephrine) on ChT using (OCT), and showed that the subfoveal choroidal thickness was significantly decreased after drop instillation which is confident with our findings. [10] Recently, performed a study examining the effect of the 3 commonly used mydriatics upon the choroidal thickness and its relationship with anterior segment parameters in 120 eyes of 240 healthy adult subjects using (OCT), and proved that the 3 drops cause choroidal thinning with an increase in volume and depth of anterior chamber, which is supporting our research data [13].

A study carried out by BP Sander, et al. [14] studying the effect of $2 \%$ homatropine (parasympatholytic) and $2.5 \%$ phenylephrine (sympathomimetic) upon the ChT in 40 eyes of healthy adult subjects revealed that the instillation of $2 \%$ homatropine resulted in a small but significant increase in subfoveal and parafoveal ChT however no significant changes in ChT were observed after $2.5 \%$ phenylephrine instillation, which is inconsistent with our results (small sample size \& different age group) [14].

However, another study done by M Kim, et al. [15] on 58 healthy eyes of 29 subjects demonstrated that tropicamide and phenylephrine have no significant influence on clinical measurement of ChT, which is not in agreement with our findings (small sample size \& different ethnicity) [15].

\section{Conclusion}

In conclusion, results succeeded to evaluate the effects of mydriatics on choroidal thickness and anterior chamber parameters.

\section{Acknowledgement}

None.

\section{Conflicts of Interest}

The authors declare no potential conflicts of interest with respect to the authorship, and/or publication of this article.

\section{References}

1. SW Cheung, R Chan, RCS Cheng, P Cho (2009) Effect of cycloplegia on axial length and anterior chamber depth measurements in children. Clinical and Experimental Optometry 92(6): 476-481.

2. F Aptel, P Denis (2010) Optical coherence tomography quantitative analysis of iris volume changes after pharmacologic mydriasis. Ophthalmology 117(1): 3-10.

3. AP Ribeiro, RM Crivelaro, PP Teixeira, DY Trujillo, PJ Guimaraes, et al. (2014) Effects of different mydriatics on intraocular pressure, pupil diameter, and ruminal and intestinal motility in healthy sheep. Vet Ophthalmol 17(6): 397-402.

4. DL Nickla, J Wallman (2010) The multifunctional choroid. Prog Retin Eye Res 29(2): 144-168. 
5. E Ahmed (2011) Comprehensive manual of ophthalmology (1st ed.) New Delhi: Jaypee Brothers.

6. RF Spaide (2009) Enhanced depth imaging optical coherence tomography of retinal pigment epithelial detachment in age related macular degeneration. Am J Ophthalmol 147(4): 644-652.

7. JC Mwanza, FE Sayyad, MR Banitt, DL Budenz (2013) Effect of pupil dilation on macular choroidal thickness measured with spectral domain optical coherence tomography in normal and glaucomatous eyes International Ophthalmology 33(4): 335-341.

8. T Fujiwara, Y Imamura, R Margolis, JS Slakter, RF Spaide (2009) Enhanced depth imaging optical coherence tomography of the choroid in highly myopic eyes. Am j Ophthalmol 148(3): 445-450.

9. I Maruko, T Iida, Y Sugano, A Ojima, T Sekiryu (2011) Subfoveal choroidal thickness in fellow eyes of patients with central serous chorioretinopathy. Retina 31(8): 1603-1608.

10. N Kara, A Demirok, G Karatas, A Basci, G Tatar Demircan A et al. (2014) Effects of two commonly used mydriatics on choroidal thickness: direct and crossover effects. j Ocul Pharmacol Ther 30(4): 366-370.
11. Jain R, Grewal S (2009) Pentacam: Principle and Clinical Applications. Current Journal of Glaucoma Practice with DVD: 20-32.

12.3D OCT-2000 Spectral Domain OCT | Topcon Medical Systems, Inc. (2016). Topconmedical.com.

13. I Yuvacı, E Pangal, S Yuvacı, N Bayram, M Ataş et al (2015) An Evaluation of Effects of Different Mydriatics on Choroidal Thickness by Examining Anterior Chamber Parameters: The Scheimpflug Imaging and Enhanced Depth Imaging-OCT Study. J Ophthalmol 2015(6): 981274.

14. BP Sander, MJ Collins, SA (2014) Read The effect of topical adrenergic and anticholinergic agents on the choroidal thickness of young health adults. Exp Eye Res 128:181-189.

15. M Kim, HJ Kwon, SC Lee (2012) Influence of mydriatics on choroidal thickness measurement using enhanced depth imaging-OCT. Optom Vis Sci 89(8): 1150-1155. 